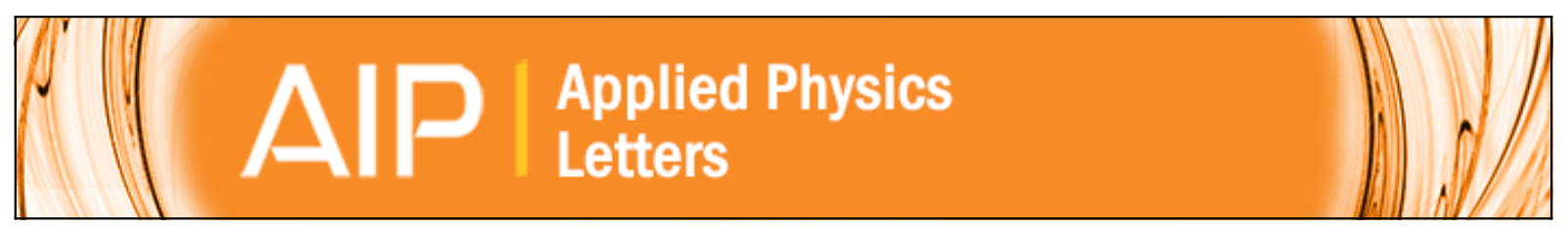

\title{
Determination of operating parameters for a GaAs-based polariton laser
}

J. Schmutzler, F. Veit, M. Aßmann, J.-S. Tempel, S. Höfling, M. Kamp, A. Forchel, and M. Bayer

Citation: Applied Physics Letters 102, 081115 (2013); doi: 10.1063/1.4794144

View online: http://dx.doi.org/10.1063/1.4794144

View Table of Contents: http://scitation.aip.org/content/aip/journal/apl/102/8?ver=pdfcov

Published by the AIP Publishing

$\stackrel{A}{A} \mathbb{P} P$ Re-register for Table of Content Alerts

Create a profile.

Sign up today! 


\title{
Determination of operating parameters for a GaAs-based polariton laser
}

\author{
J. Schmutzler, ${ }^{1}$ F. Veit, ${ }^{1}$ M. Aßmann, ${ }^{1}$ J.-S. Tempel, ${ }^{1}$ S. Höfling, ${ }^{2}$ M. Kamp, ${ }^{2}$ \\ A. Forchel, ${ }^{2}$ and M. Bayer ${ }^{1}$ \\ ${ }^{1}$ Experimentelle Physik 2, Technische Universität Dortmund, D-44221 Dortmund, Germany \\ ${ }^{2}$ Technische Physik, Physikalisches Institut, Wilhelm Conrad Röntgen Research Center \\ for Complex Material Systems, Universität Würzburg, D-97074 Würzburg, Germany
}

(Received 28 November 2012; accepted 18 February 2013; published online 28 February 2013)

\begin{abstract}
We report on a systematic study of the phase transitions to polariton condensation (PC) and further to cavity lasing in a GaAs-based microcavity with respect to exciton-cavity detuning and lattice temperature. Using far field and time-resolved spectroscopy, we determined the parameter space in which PC can be achieved and the corresponding variation of PC threshold power. We found a lower bound of $-12 \mathrm{meV}$ for the exciton-cavity detuning and an upper bound of $90 \mathrm{~K}$ for the lattice temperature. (ㅇ 2013 American Institute of Physics. [http://dx.doi.org/10.1063/1.4794144]
\end{abstract}

Since the observation of the so-called strong coupling regime in semiconductor microcavities, ${ }^{1}$ the field of semiconductor based cavity quantum electrodynamics has been intensively studied due to many promising applications such as inversionless polariton lasers ${ }^{2}$ or building blocks for all-optical logic circuits. ${ }^{3-6}$ In the last years, Bose-Einstein condensation (BEC) of exciton-polaritons, which is a prerequisite for a polariton laser device, was reported for different material systems such as $\mathrm{CdTe},{ }^{7} \mathrm{ZnO},{ }^{8} \mathrm{GaN},{ }^{9}$ and $\mathrm{GaAs} .{ }^{10}$

On the road towards a polariton laser, systematic studies need to be done in order to determine the optimal operating parameters. Detailed investigations of the threshold power dependence on the lattice temperature and the exciton-cavity detuning for GaN-based ${ }^{11,12}$ and CdTe-based ${ }^{13,14}$ microcavities were accomplished. However, for GaAs-based microcavities, there exist only studies for one single detuning ${ }^{15}$ or for a limited range of temperatures and detunings. ${ }^{16}$ Moreover, there is an ongoing debate about the distinction between polariton condensation (PC) and cavity lasing $(\mathrm{CL})^{17,18}$ and the identification of unique criteria for PC is still in the focus of current studies. ${ }^{19,20}$ An interpretation of coherent emission in the weak coupling regime in terms of a photon BEC has also been suggested. ${ }^{21}$

In this contribution, we present a systematic investigation of the phase transitions to PC and CL for a wide range of temperatures and detunings in a GaAs-based microcavity. Thereby, we determined an upper limit of temperature of $90 \mathrm{~K}$ and a lower limit of $\delta=E_{c}-E_{x}=-12 \mathrm{meV}$ of detuning for PC in our sample, where $E_{c}\left(E_{x}\right)$ denotes the energy of the cavity (exciton).

We investigated a GaAs-based $\lambda / 2$-microcavity with a Rabi splitting of about $14 \mathrm{meV}$ and a quality factor of about 1800 (further details on the sample are given in Refs. 15 and 20). The sample was mounted in a helium-flow cryostat, measurements were performed in a temperature range of $10-110 \mathrm{~K}$. For optical excitation, a picosecond-pulsed titanium-sapphire laser (repetition rate $75.39 \mathrm{MHz}$ ) was tuned to the first sideband minimum of the distributed Bragg reflector (DBR) stopband at about $1666 \mathrm{meV}$. The laser beam was focused under $45^{\circ}$ of incidence onto the sample, the shape of the spot was Gaussian and about $30 \mu \mathrm{m}$ in diameter.
The emission from the sample was collected using a microscope objective (numerical aperture 0.26); the far field emission was studied by imaging the Fourier plane of the objective onto the entrance slit of a monochromator. For detection, a liquid nitrogen-cooled CCD-camera was used. Time-resolved measurements were accomplished with a streak camera (time resolution 3-4 ps). Here, spectral resolution was provided by a liquid crystal filter (full width at half maximum $0.7 \mathrm{~nm}$ ) positioned in front of the streak camera.

Figs. 1 and 2 show example far field emission images for different excitation power levels P for detunings of $\delta=$ $-7.0 \mathrm{meV}$ and $\delta=7.7 \mathrm{meV}$ at $T=10 \mathrm{~K}$. For the negative detuning case, one observes a blue shift of $1.9 \mathrm{meV}$ with respect to the minimum of the lower polariton (LP) branch at the phase transition to PC $\left[P=P_{t h r}\right.$, Fig. 1(b)]. At high excitation power, two different modes occur [Fig. 1(d)]. We attribute the low energy mode to PC and the high energy mode to bare CL, which is in good agreement with the calculated dispersion. The occurrence of strong and weak coupling regimes is due to carrier density changing during one excitation pulse (see also Fig. 3). In contrast, for the positive detuning case, a rather large blue shift of $13.8 \mathrm{meV}$ at the phase transition to PC is observed [Fig. 2(b)]. Surprisingly, PC occurs at higher energies than the expected cavity mode. At slightly higher excitation power of $P=1.56 P_{t h r}$, one further mode appears at an energy even above the upper polariton branch [Fig. 2(c)].

For this experimental setting, we have performed an additional streak camera measurement [Fig. 3]. The high energy peak exhibits a pulse duration (full width at half maximum) of about 6 ps. After the decay of the high energy peak, the low energy peak rises with a pulse duration of about 15 ps. We construe this observation as follows: After the decrease of the carrier density, a transition back to the strong coupling regime takes place; the bare CL breaks down and PC arises. Thus, the high energy peak can be attributed to bare CL, the low energy peak to PC. A transition from weak to strong coupling regime during one excitation pulse was also observed previously. ${ }^{21}$ For high excitation power, only a single mode is observed [Fig. 2(d)], which we attribute to CL. 


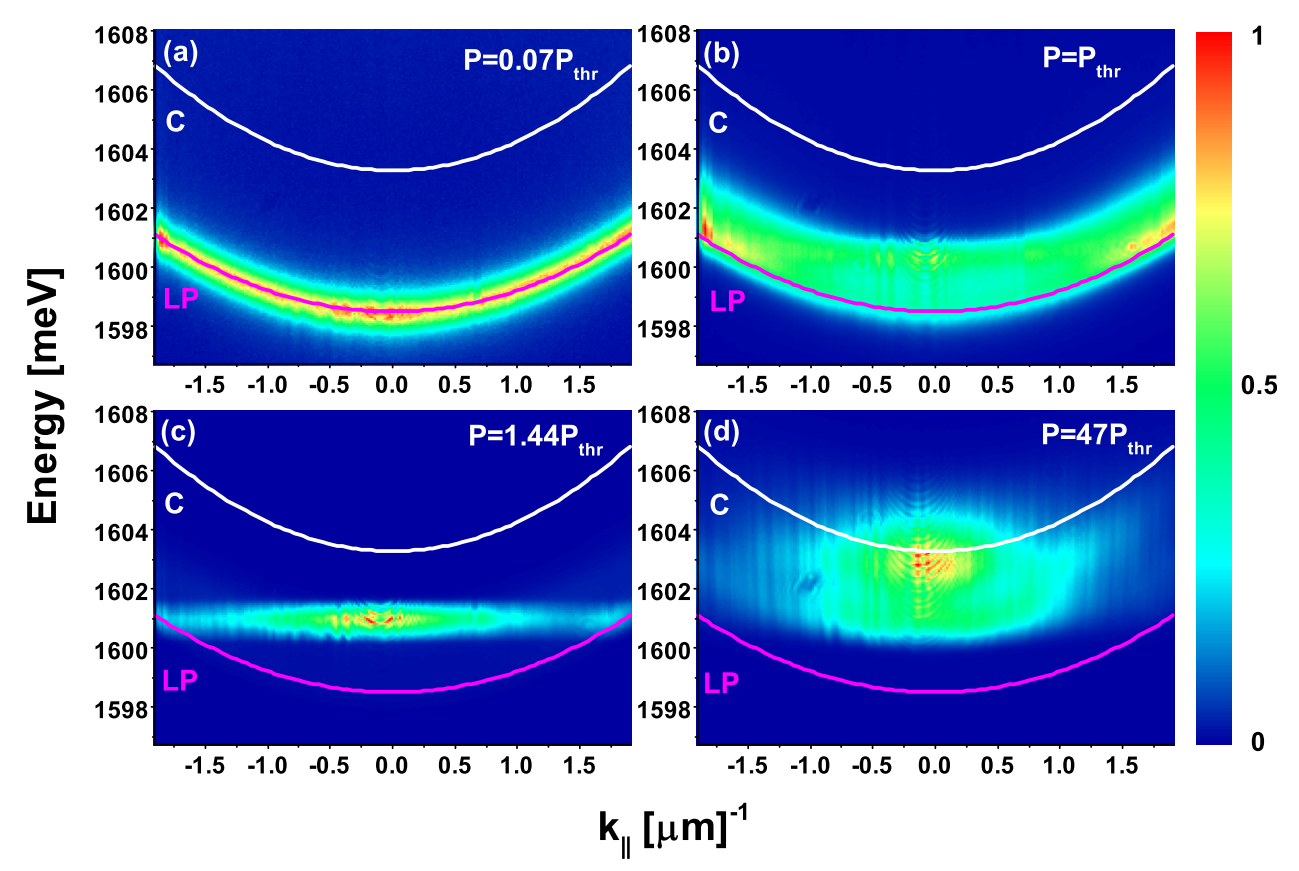

FIG. 1. Far field emission for different excitation power levels at $\delta=-7.0 \mathrm{meV}$. The solid lines are calculated curves. C, cavity dispersion; LP, lower polariton dispersion.

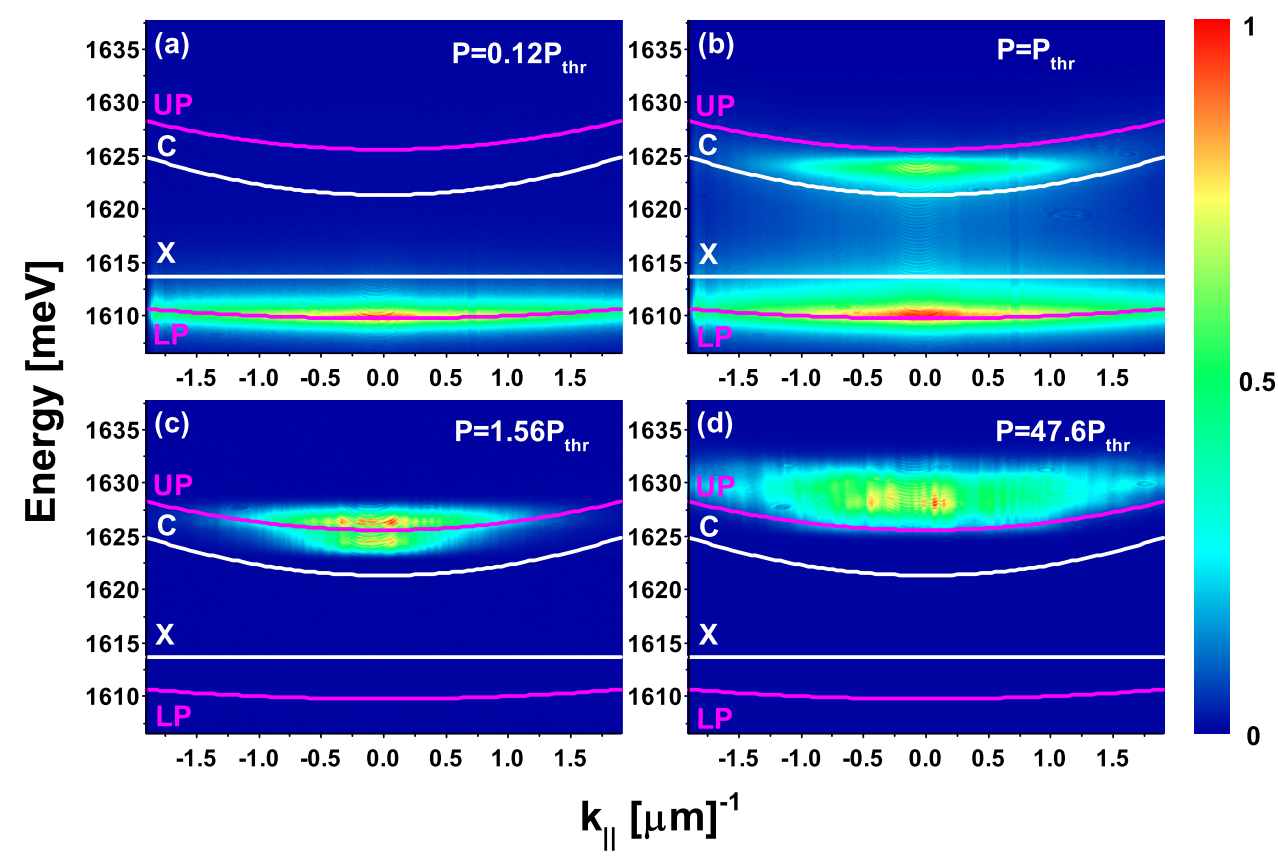

FIG. 2. Far field emission for different excitation power levels at $\delta=7.7 \mathrm{meV}$. The solid lines are calculated curves. C, cavity dispersion; LP, lower polariton dispersion; UP, upper polariton dispersion; $\mathrm{X}$, exciton dispersion.

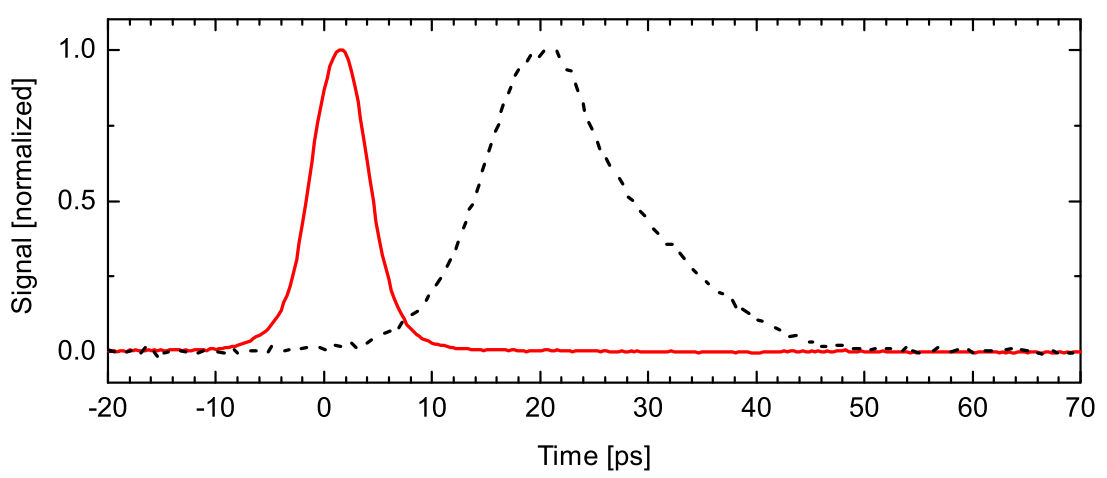

FIG. 3. Time-resolved emission at $\delta=7.7 \mathrm{meV}$, $T=10 \mathrm{~K}$, and $P=1.56 P_{t h r}$. Spectral sensitivity is provided by a tunable liquid crystal filter. Red line, $E=1626.9 \mathrm{meV}$; black dashed line, $E=1624.0 \mathrm{meV}$.

Fig. 4 shows the detuning dependent emission energies (in-plane momentum $k_{\|}=0$ ) at $T=10 \mathrm{~K}$ and $T=90 \mathrm{~K}$ for low power excitation, excitation at threshold and $P>10 P_{t h r}$. At threshold for $T=10 \mathrm{~K}$, a significant blueshift varying from 1.5 to $13.8 \mathrm{meV}$ relative to the minimum of the LP branch occurs [Fig. 4(a)]. The highest blueshift is observed at $\delta=7.7 \mathrm{meV}$, the lowest at $\delta=-6.3 \mathrm{meV}$. For $\delta>5 \mathrm{meV}$, the emission at threshold is even higher in energy than the expected cavity 


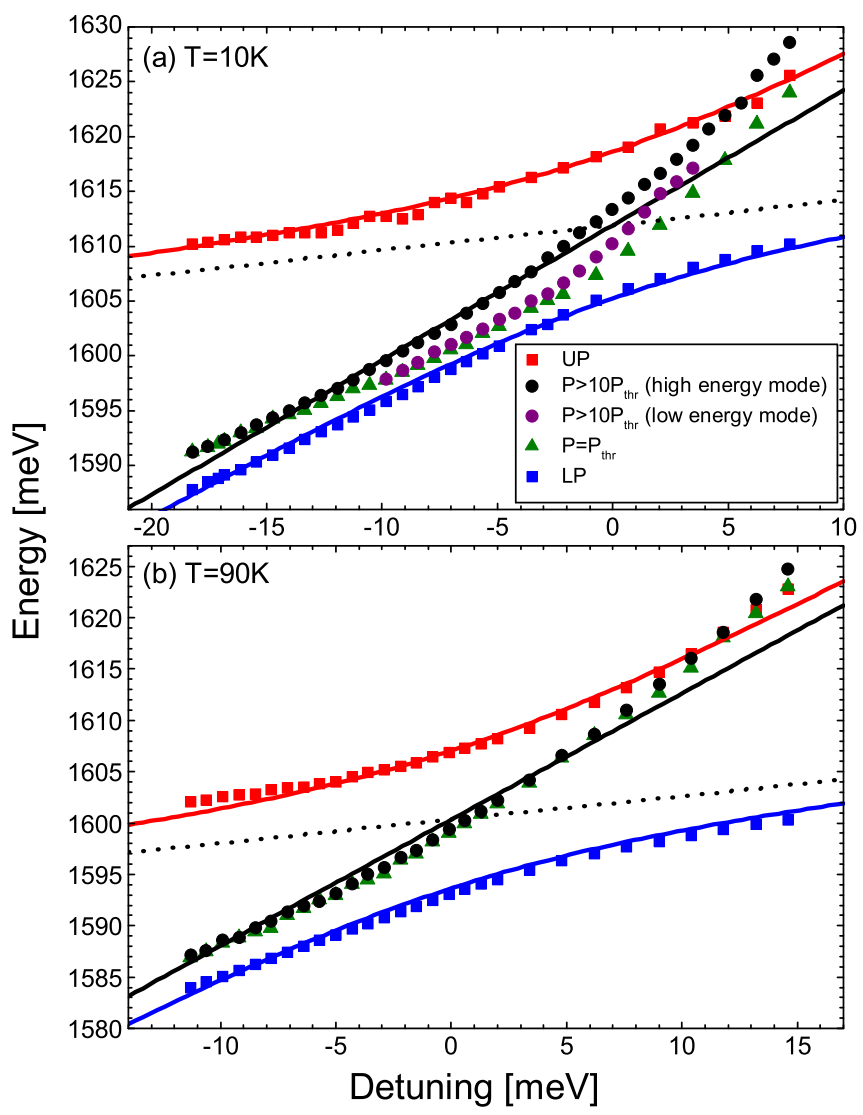

FIG. 4. Detuning dependent emission $\left(k_{\|}=0\right)$ at $T=10 \mathrm{~K}$ and $T=90 \mathrm{~K}$. Solid blue (red) line, lower (upper) polariton branch fitted to the data; black solid line, expected cavity mode; black dotted line, expected exciton energy. Note that for $T=10 \mathrm{~K}$, two mode emission is not observed for several positive detunings at high power excitation $P>10 P_{t h r}$, but for intermediate excitation power (typically $P<10 P_{t h r}$ ).

mode. At strong negative detuning $\delta<-12 \mathrm{meV}$, the emission lies approximately at the expected cavity mode. In the case of high excitation power, two emission modes can be seen in a range of $\delta=[-10 ; 3] \mathrm{meV}$ as discussed in detail above for the case of $\delta=-7.0 \mathrm{meV}$ [Fig. 1(d)].

Time-resolved measurements reveal the same results as shown in Fig. 3. The high energy peak has a pulse duration of about 6-7 ps and is followed in time by the low energy peak with a pulse duration of about $15-30 \mathrm{ps}$. We, therefore, attribute the high energy mode to pure CL and the low energy mode to PC. Also for $\delta>3 \mathrm{meV}$, two mode emission can be found, but only in the case of intermediate excitation power (typically $P<10 P_{t h r}$, [Fig. 2(c)]). For $\delta<-13 \mathrm{meV}$, no significant shift in energy can be observed when increasing the excitation power from $P=P_{t h r}$ to $P>10 P_{t h r}$. This can be explained by a direct transition to $\mathrm{CL}$ without occurrence of $\mathrm{PC}$.

From these findings, we conclude three criteria that need to be fulfilled to observe a phase transition to PC: (i) emission energy $E<E_{c}$ at threshold for negative detuning, (ii) occurrence of two modes for excitation power levels above threshold in pulsed regime, and (iii) further blueshift of the emission when increasing the excitation power far above threshold. According to these criteria, PC can be observed in a range of $\delta=[-9.8 ; 7.7] \mathrm{meV}$ for $T=10 \mathrm{~K}$ [Fig. 4(a)], $\delta=[-12.0 ; 8.3] \mathrm{meV}$ for $T=30 \mathrm{~K}, \delta=[-10.3 ; 9.3] \mathrm{meV}$ for $T=50 \mathrm{~K}$, and $\delta=[-8.0 ; 11.6] \mathrm{meV}$ for $T=70 \mathrm{~K} .{ }^{22}$ Note, however, that the upper $\delta$-bound is determined by our sample edge that does not allow larger positive detunings. Apparently, there exists a lower bound for PC with respect to detuning for the investigated structure when reaching a negative detuning in the order of magnitude of the Rabi splitting. Possibly, a reduced carrier scattering cross section due to high photonic fraction prohibits the formation of PC. This finding coincides with earlier measurements at quasi-resonant excitation in which a lower bound for PC was observed at $\delta=-10 \mathrm{meV} .^{23}$ At temperatures from $T=90 \mathrm{~K}$, no phase transition to $\mathrm{PC}$ occurs since there is only one mode observable and no significant further blueshift can be seen when increasing the excitation power far above threshold [Fig. 4(b)]. ${ }^{22}$ This finding is in good agreement with earlier results at $\delta=0 \mathrm{meV}^{15}$

For all temperatures, a prominent blueshift of CL with respect to the expected cavity mode for positive detunings is determined. This seems to contradict studies in which a redshift of the cavity mode was reported. ${ }^{17,18}$ However, in these studies, continuous wave lasers were used which create a rather equilibrium background carrier distribution in contrast
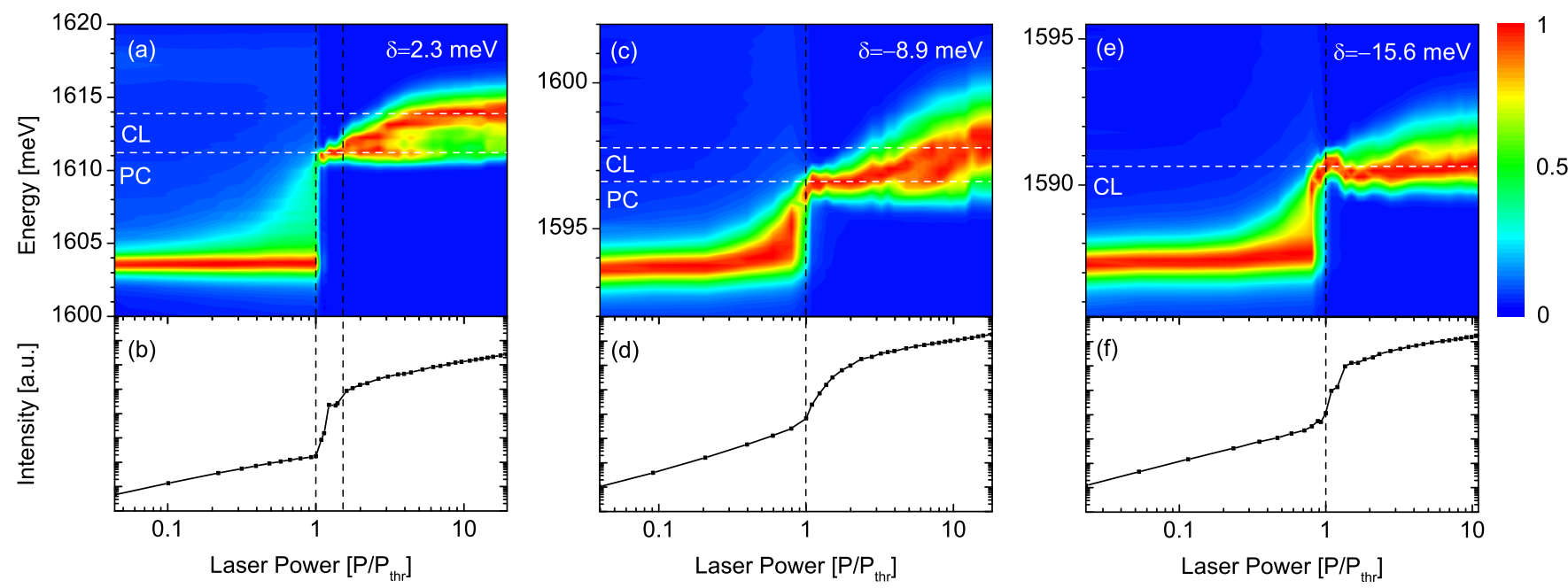

FIG. 5. Power dependent spectra (in-plane momentum $\left|k_{\|}\right|<0.085 \mu \mathrm{m}^{-1}$ ) and corresponding I-O curves for three different detunings $\delta=2.3 \mathrm{meV}$, $\delta=-8.9 \mathrm{meV}$, and $\delta=-15.6 \mathrm{meV}$ at $T=50 \mathrm{~K}$. Modes of emission are indicated by white dashed lines and thresholds in the I-O curves are indicated by black dashed lines. Laser power is normalized to the first threshold power $P_{t h r}$. Note: Power dependent spectra are normalized to one for each laser power separately. 
to our experimental situation. We note that a blueshift of the cavity mode with increasing carrier density was reported by another group at negative detuning. ${ }^{24}$

In addition, we investigated the dependence of the emission on the excitation power. Fig. 5 shows example inputoutput (I-O) curves and the corresponding power dependent spectra (in-plane momentum $\left|k_{\|}\right|<0.085 \mu \mathrm{m}^{-1}$ ) at three different detunings. For detunings of $\delta=2.3 \mathrm{meV}$ and $\delta$ $=-8.9 \mathrm{meV}$, two modes of emission and an additional blueshift when increasing the laser power above threshold can clearly be seen [Figs. 5(a) and 5(c)]. In contrast, for a large negative detuning of $\delta=-15.6 \mathrm{meV}$, only one mode of emission without additional blueshift is observed [Fig. 5(e)]. Therefore, we conclude that for $\delta=2.3 \mathrm{meV}$ and $\delta=$ $-8.9 \mathrm{meV}$ PC can be achieved, whereas in the latter case only CL occurs. Earlier, there was a claim that the observation of a two-threshold behavior in the I-O curve is a prerequisite for the occurrence of PC. ${ }^{17}$ According to our data, this is not necessarily the case: Whereas there is evidence for two thresholds in the I-O curve in the case of $\delta=2.3 \mathrm{meV}$ [Fig. 5(b)], only a single threshold occurs for $\delta=-8.9 \mathrm{meV}$ [Fig. 5(d)]. From previous reports, it is well known that a two-threshold behavior in the I-O curve can be very pronounced in further confined structures as nanowires ${ }^{25}$ and micropillars. ${ }^{26}$ However, there are only a few publications in which such an effect was observed in planar microcavities. ${ }^{15,20,27}$ Here, we want to emphasize that the two-threshold criterion with respect to the I-O curve might be sufficient but it is not a prerequisite for the observation of PC.

Fig. 6 shows the threshold power dependence on detuning at different temperatures. The lowest threshold is observed for $T=10 \mathrm{~K}$ and $\delta \approx 6 \mathrm{meV}$. When going to negative detuning, the $\mathrm{PC}$ threshold power increases due to less efficient scattering into the $k_{\|}=0$-state and shortened LP lifetime. On the other hand, when going to positive detuning the effective mass rises due to higher excitonic fraction of the LP and therefore the threshold increases again for $\delta>6 \mathrm{meV}$.

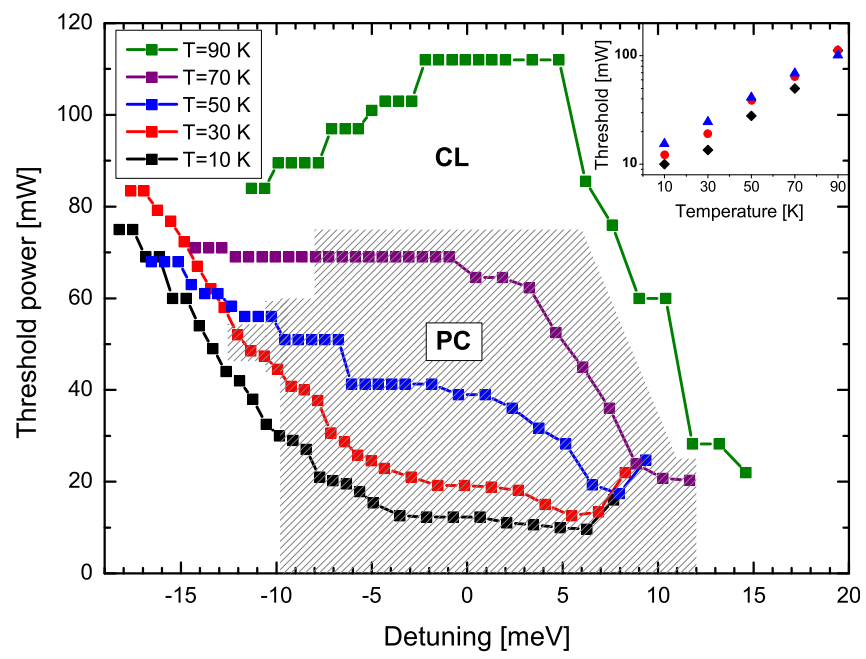

FIG. 6. Dependence of threshold power on detuning for different lattice temperatures. Data points inside (outside) the shaded area are attributed to PC (CL). Inset: Dependence of threshold power on lattice temperature for different detunings. Black diamonds, $\delta=5 \mathrm{meV}$; red circles, $\delta=0 \mathrm{meV}$; and blue triangles, $\delta=-5 \mathrm{meV}$.
Interestingly, there is no sharp increase of the threshold power observable when going from strong to weak coupling regime around $\delta=[-12 ;-8] \mathrm{meV}$ in the temperature range of $T=10-70 \mathrm{~K}$. The inset of Fig. 6 shows the dependence of threshold power on lattice temperature for three different detunings $(\delta=-5 \mathrm{meV}, \delta=0 \mathrm{meV}$, and $\delta=5 \mathrm{meV})$, in which PC can be achieved up to $T=70 \mathrm{~K}$. While there is a systematic rise of threshold with increasing temperature, there is no significant jump observable when going from strong coupling regime at $T=70 \mathrm{~K}$ to weak coupling regime at $T=90 \mathrm{~K}$. A rather small increase of threshold, namely a doubling, was recently reported in a high-finesse GaAs-based microcavity for $\delta=-5 \mathrm{meV}$ when going from strong to weak coupling regime in a temperature range of $T=25-70 \mathrm{~K} .{ }^{27}$ For a similar configuration $(\delta=-5 \mathrm{meV}, T=30-90 \mathrm{~K})$, we observe an increase by a factor of 4 .

In conclusion, we determined precise parameters with respect to exciton-cavity detuning and sample temperature at which PC can be observed. A lower limit of $-12 \mathrm{meV}$ for detuning and an upper temperature of $90 \mathrm{~K}$ was found for PC. However, the observation of two thresholds in the I-O curve is not a prerequisite for the occurrence of PC in this study.

The Dortmund group acknowledges support from the Deutsche Forschungsgemeinschaft through the research grants DFG 1549/18-1 and 1549/19-1. The group at Würzburg University acknowledges support from the State of Bavaria.

${ }^{1}$ C. Weisbuch, M. Nishioka, A. Ishikawa, and Y. Arakawa, Phys. Rev. Lett. 69, 3314 (1992).

${ }^{2}$ A. Imamoğlu, R. J. Ram, S. Pau, and Y. Yamamoto, Phys. Rev. A 53, 4250 (1996).

${ }^{3}$ C. Leyder, M. Romanelli, J. P. Karr, E. Giacobino, T. C. H. Liew, M. M. Glazov, A. V. Kavokin, G. Malpuech, and A. Bramati, Nat. Phys. 3, 628 (2007).

${ }^{4}$ D. Sarkar, S. S. Gavrilov, M. Sich, J. H. Quilter, R. A. Bradley, N. A. Gippius, K. Guda, V. D. Kulakovskii, M. S. Skolnick, and D. N. Krizhanovskii, Phys. Rev. Lett. 105, 216402 (2010).

${ }^{5}$ C. Adrados, A. Amo, T. C. H. Liew, R. Hivet, R. Houdré, E. Giacobino, A. V. Kavokin, and A. Bramati, Phys. Rev. Lett. 105, 216403 (2010).

${ }^{6}$ A. Amo, S. Pigeon, C. Adrados, R. Houdré, E. Giacobino, C. Ciuti, and A. Bramati, Phys. Rev. B 82, 081301 (2010).

${ }^{7}$ J. Kasprzak, M. Richard, S. Kundermann, A. Baas, P. Jeambrun, J. M. Keeling, F. Marchetti, M. Szymańska, R. André, J. Staehli, V. Savona, P. B. Littlewood, B. Deveaud, and L. S. Dang, Nature 443, 409 (2006).

${ }^{8}$ H. Franke, C. Sturm, R. Schmidt-Grund, G. Wagner, and M. Grundmann, New J. Phys. 14, 013037 (2012).

${ }^{9}$ S. Christopoulos, G. Baldassarri Höger von Högersthal, A. J. D. Grundy, P. G. Lagoudakis, A. V. Kavokin, J. J. Baumberg, G. Christmann, R. Butté, E. Feltin, J.-F. Carlin, and N. Grandjean, Phys. Rev. Lett. 98, 126405 (2007).

${ }^{10}$ R. Balili, V. Hartwell, D. Snoke, L. Pfeiffer, and K. West, Science 316, 1007 (2007).

${ }^{11}$ R. Butté, J. Levrat, G. Christmann, E. Feltin, J. F. Carlin, and N. Grandjean, Phys. Rev. B 80, 233301 (2009).

${ }^{12}$ J. Levrat, R. Butté, E. Feltin, J. F. Carlin, N. Grandjean, D. Solnyshkov, and G. Malpuech, Phys. Rev. B 81, 125305 (2010).

${ }^{13}$ J. Kasprzak, M. Richard, A. Baas, B. Deveaud, R. André, J.-P. Poizat, and L. S. Dang, Phys. Rev. Lett. 100, 067402 (2008).

${ }^{14}$ F. M. Marchetti, M. H. Szymańska, J. M. J. Keeling, J. Kasprzak, R. André, P. B. Littlewood, and L. Si Dang, Phys. Rev. B 77, 235313 (2008).

${ }^{15}$ J.-S. Tempel, F. Veit, M. Aßmann, L. E. Kreilkamp, S. Höfling, M. Kamp, A. Forchel, and M. Bayer, New J. Phys. 14, 083014 (2012).

${ }^{16}$ E. Wertz, L. Ferrier, D. D. Solnyshkov, P. Senellart, D. Bajoni, A. Miard, A. Lemaitre, G. Malpuech, and J. Bloch, Appl. Phys. Lett. 95, 051108 (2009).

${ }^{17}$ D. Bajoni, P. Senellart, A. Lemaitre, and J. Bloch, Phys. Rev. B 76, 201305 (2007). 
${ }^{18}$ R. Balili, B. Nelsen, D. W. Snoke, L. Pfeiffer, and K. West, Phys. Rev. B 79, 075319 (2009).

${ }^{19}$ A. Rahimi-Iman, A. V. Chernenko, J. Fischer, S. Brodbeck, M. Amthor, C. Schneider, A. Forchel, S. Höfling, S. Reitzenstein, and M. Kamp, Phys. Rev. B 86, 155308 (2012).

${ }^{20}$ J.-S. Tempel, F. Veit, M. Aßmann, L. E. Kreilkamp, A. Rahimi-Iman, A. Löffler, S. Höfling, S. Reitzenstein, L. Worschech, A. Forchel, and M. Bayer, Phys. Rev. B 85, 075318 (2012).

${ }^{21}$ E. Kammann, H. Ohadi, M. Maragkou, A. V. Kavokin, and P. G. Lagoudakis, New J. Phys. 14, 105003 (2012).

${ }^{22}$ See supplementary material at http://dx.doi.org/10.1063/1.4794144 for data at $30 \mathrm{~K}, 50 \mathrm{~K}, 70 \mathrm{~K}$, and $110 \mathrm{~K}$.
${ }^{23}$ M. Aßmann, J.-S. Tempel, F. Veit, M. Bayer, A. Rahimi-Iman, A. Löffler, S. Höfling, S. Reitzenstein, L. Worschech, and A. Forchel, Proc. Natl. Acad. Sci. U.S.A. 108, 1804 (2011).

${ }^{24}$ G. Christmann, G. Tosi, N. G. Berloff, P. Tsotsis, P. S. Eldridge, Z. Hatzopoulos, P. G. Savvidis, and J. J. Baumberg, Phys. Rev. B 85, 235303 (2012).

${ }^{25}$ A. Das, J. Heo, M. Jankowski, W. Guo, L. Zhang, H. Deng, and P. Bhattacharya, Phys. Rev. Lett. 107, 066405 (2011).

${ }^{26}$ D. Bajoni, E. Semenova, A. Lemaître, S. Bouchoule, E. Wertz, P. Senellart, and J. Bloch, Phys. Rev. B 77, 113303 (2008).

${ }^{27}$ P. Tsotsis, P. S. Eldridge, T. Gao, S. I. Tsintzos, Z. Hatzopoulos, and P. G. Savvidis, New J. Phys. 14, 023060 (2012). 